\title{
Myelodysplastic syndrome
}

\section{Richard A. Wells MD DPhil, Rena Buckstein MD, Jeremy Rezmovitz MD}

\section{Myelodysplastic syndrome is a disease of older adults and is becoming more common}

Myelodysplastic syndrome, an acquired clonal disorder of the bone marrow, is a common cause of unexplained anemia in North American adults older than 65 years. ${ }^{1}$ Most cases are idiopathic, although some are related to prior chemotherapy or radiotherapy. Men are affected more commonly than women. The median age at diagnosis is 76 years, and the incidence rises to 36.4 cases/100 000 population per year in those over 80 years. $^{2}$ As the population ages, rates will increase.
Myelodysplastic syndrome should be suspected in older patients with macrocytic anemia

If macrocytic anemia develops in patients older than 50 years, myelodysplastic syndrome should be considered, especially in the presence of reduced platelet or leukocyte count. Diagnosis requires bone marrow examination, including cytogenetic testing; however, five clinical clues to the diagnosis are age over 65 years, presence of macrocytic anemia, presence of other types of cytopenia, increased red blood cell distribution width and increased lactate dehydrogenase level. ${ }^{4}$

\section{Patients with unexplained severe or progressive cytopenia should be referred to a hematologist}

If patients are diagnosed with myelodysplastic syndrome, prognostic classification, based on depth of cytopenia, bone marrow blast count and cytogenetic variables, allows them to be assigned to risk categories ranging from very low risk (median survival $8.8 \mathrm{yr}$ ) to very high risk (median survival $0.8 \mathrm{yr}){ }^{5}$

\section{References}

1. Guralnik JM, Eisenstaedt RS, Ferrucci L, et al. Prevalence of anemia in persons 65 years and older in the United States: evidence for a high rate of unexplained anemia. Blood 2004;104:2263-8.

2. Rollison DE, Howlader N, Smith MT, et al. Epidemiology of myelodysplastic syndromes and chronic myeloproliferative disorders in the United States, 2001-2004, using data from the NAACCR and SEER programs. Blood 2008;112:45-52

3. Lindquist KJ, Danese MD, Mikhael J, et al. Health care utilization and mortality among elderly patients with myelodysplastic syndromes. Ann Oncol 2011; 22:1181-8.
4. Rauw J, Wells RA, Chesney A, et al. Validation of a scoring system to establish the probability of myelodysplastic syndrome in patients with unexplained cytopenias or macrocytosis. Leuk Res 2011;35:1335-8.

5. Greenberg PL, Tuechler H, Schanz J, et al. Revised international prognostic scoring system for myelodysplastic syndromes. Blood 2012;120: 2454-65.

6. Killick SB, Carter C, Culligan D, et al.; British Committee for Standards in Haematology. Guidelines for the diagnosis and management of adult myelodysplastic syndromes. Br J Haematol 2014; 164:503-25.
An interactive decision tool for the investigation and management of active treatment for myelodysplastic syndrome is available at www.MDSclearpath.org. The development of this tool was funded by Celgene Canada.

\section{Myelodysplastic syndrome com- monly presents as fatigue}

Patients usually present with symptoms of anemia (e.g., fatigue, dyspnea), but some may have bruising, bleeding or petechiae (from thrombocytopenia) or recurrent infections (from neutropenia). Without active treatment, most patients will eventually require red blood cell transfusions.

Active treatment is available and improves outcomes in myelodysplastic syndrome

Younger patients may qualify for stem cell transplantation. Drug therapies such as lenalidomide (taken orally), erythropoietin (administered by subcutaneous injections at home) and azacitidine (administered by subcutaneous injections at cancer clinics) do not cure myelodysplastic syndrome but can reduce transfusion dependency, improve quality of life and extend survival. ${ }^{5,6}$

Competing interests: Richard Wells declare grants and personal fees from Novartis Canada and Celgene Canada, and personal fees from Janssen. Rena Buckstein declares research support and honoraria from Celgene and honoraria from Novartis. No other competing interests were declared.

This article has been peer reviewed.

Affiliations: Division of Medical Oncology and Hematology, Odette Cancer Centre (Wells, Buckstein); Department of Family Medicine (Rezmovitz), Sunnybrook Health Sciences Centre, Toronto, Ont.

Correspondence to: Richard Wells, richard. wells@sunnybrook.ca

CMAJ 2016. DOI:10.1503/cmaj.151077 